\title{
EL ESTUDIO DE LAS PRÁCTICAS EDUCATIVAS Y SU RELEVANCIA PARA EL ANÁLISIS DE PROCESOS DE FORMACIÓN EN DOCENCIA UNIVERSITARIA $^{1}$
}

\section{Jorge Chávez y Claudia Jaramillo²}

\section{RESUMEN}

El presente trabajo tiene por objetivo presentar un modelo teórico y metodológico que permita abordar los procesos de formación en docencia universitaria desde una perspectiva teórica de orientación sociocultural. Esta considera al aprendizaje como una construcción social de significados y a la enseñanza como un proceso de ayuda planificada, sistemática y sostenida para dicha elaboración. En este contexto, es de especial relevancia proporcionar un modelo que permita la descripción y la comprensión de la organización de los diferentes componentes que debe incluir la formación en docencia universitaria, dados los distintos esfuerzos que realizan las universidades para ubicar en el centro de la actividad académica la enseñanza y el aprendizaje.

Palabras clave: práctica educativa, formación en docencia universitaria, análisis de interacción, perspectiva sociocultural.

\section{THE STUDY OF EDUCATIONAL PRACTICES AND THEIR RELEVANCY FOR THE ANALYSIS OF UNIVERSITY TEACHING PREPARATION PROCESSES.}

\begin{abstract}
This article aims to present a theoretical and methodological framework that allows for a better understanding of the training processes in university teaching, from a theoretical perspective of a sociocultural orientation. This perspective considers learning as a social construction of meanings and teaching as a process of planned, systematic and sustained support for this construction process. In this context, it is of particular relevance to provide a framework that allows for the description and understanding of the organization of the different components that should be included in the preparation of university professors, given the efforts made by universities to make the processes of teaching and learning the center of their academic activities.
\end{abstract}

Keywords: educational practice, university teaching preparation, analysis of interaction, sociocultural perspective

1 Este trabajo se enmarca en el proyecto "Desarrollo Académico en Investigación e Innovación Educativa para mejorar el aprendizaje de los estudiantes de la Universidad de Santiago de Chile". Concurso FIAC2 2011(USA 1112) del Ministerio de Educación de Chile.

2 Unidad de Innovación Educativa, Universidad de Santiago de Chile, Santiago, Chile. Contacto: jorge.chavez@usach.cl; claudia.jaramillo@usach.cl 


\section{Introducción}

Los cambios recientes en la gestión del conocimiento y en la forma de concebir el aprendizaje han impulsado la necesidad de implementar importantes innovaciones en la forma en que se enseña, no solo en educación superior, sino también en diferentes niveles y escenarios educativos (Barron, 2006). En este sentido, los programas de formación en docencia universitaria han adquirido especial relevancia a la hora de llevar a cabo mejoras en las formas de enseñanza que realizan los docentes (Postareff, Lindblom-Ylänne \& Nevgi, 2007).

En este contexto, diversas investigaciones buscan determinar el impacto de los programas formativos implementados en los resultados de aprendizaje (Ho, Watkins \& Kelly, 2001; Stes, De Maeyer, Gijbels $\&$ Van Petegem, 2013). Sin embargo, a pesar de la importancia que ha adquirido el estudio de los procesos de formación, la mayoría de las investigaciones en esta materia se han limitado a aspectos relacionados con la satisfacción de los participantes, así como al estudio de los enfoques de enseñanza-aprendizaje que adquieren docentes y estudiantes. Por este motivo, es cada vez más relevante profundizar en la forma en que estos programas se llevan a cabo, para así indagar también en el nivel de apropiación y transferencia que pueden llegar a tener en la práctica (Stes et al., 2013).

Esto ha llevado a una serie de cuestionamientos vinculados con la necesidad de profundizar en el impacto de estos procesos formativos desde una perspectiva cualitativa, incorporando el estudio de la actividad que desarrollan profesores y docentes formadores en un proceso de perfeccionamiento (Stes, Min-Leliveld, Gijbels $\&$ Van Petegem, 2010). En este contexto, creemos necesario complementar la investigación hasta ahora desarrollada con marcos referenciales que consideren el estudio de la práctica educativa como estrategia teórica y metodológica de los procesos de formación de docentes universitarios.

El estudio de la práctica educativa permite analizar cómo se materializan dichos procesos formativos, y cuáles son las formas que asume la actividad conjunta desarrollada por los docentes formadores y los docentes universitarios al interior de un proceso instruccional. 
Los marcos referenciales para el estudio de la práctica educativa los podemos encontrar en los planteamientos de Vygotsky y su evolución posterior, en enfoques como el discurso educacional, el análisis ecológico del aula y la teoría de la actividad (Cole, 1998; Coll, Onrubia y Majós, 2008; Kitayama \& Cohen, 2010).

En resumen, el trabajo que se presenta tiene como propósito facilitar una serie de elementos teóricos y metodológicos asociados al análisis del aprendizaje desde una perspectiva sociocultural, con énfasis en el estudio de la interacción educativa. Este enfoque permite abordar el estudio de los procesos de formación a partir del análisis de las prácticas educativas y, con ello, introducir nuevas perspectivas orientadas a generar investigación que pueda contribuir al mejoramiento de la práctica docente en educación superior.

\section{El estudio de las prácticas educativas}

Existe acuerdo en que los procesos de formación asociados a la enseñanza pueden considerarse un factor relevante a la hora de mejorar las prácticas educativas en docentes universitarios (Stes et al., 2013). Sin embargo, los abordajes al estudio de dichos procesos de formación pueden ser muy variados. Los elementos compartidos desde esta línea de investigación se basan principalmente en la idea que al analizar cualquier proceso de enseñanza y aprendizaje es necesario valorar los diferentes elementos o componentes que participan en dicho proceso (Coll y Sánchez, 2008). Estos elementos varían en función de la representación de aprendizaje que se considere para su análisis.

Desde la perspectiva constructivista de orientación sociocultural, el aprendizaje formal se concibe como un proceso de construcción social mediado por significados en torno a los contenidos de aprendizaje, donde la enseñanza corresponde a un proceso de ayuda planificada, sistemática y sostenida acorde a dicho proceso de construcción (Coll et al., 2008). En este sentido, esta línea de investigación se enfoca en las formas que asumen las ayudas educativas ejercidas por los profesores y los propios estudiantes mientras interactúan en una situación de enseñanza y aprendizaje 
(Coll et al., 2008). En términos generales, desde esta perspectiva teórica se entiende por "ayuda educativa" todos los apoyos prestados por el profesor a los estudiantes, en atención a sus requerimientos y necesidades, durante un proceso de enseñanza y aprendizaje.

En este marco, la propuesta de análisis que se presenta proviene de los trabajos desarrollados por Coll y colaboradores y considera cuatro elementos relevantes para el estudio de la práctica educativa. El primer aspecto está constituido por las actuaciones de los participantes que ocurren en una situación de interacción; el segundo son las actuaciones asociadas a un contenido específico, o bien, a una tarea de aprendizaje; el tercero es el tiempo en el que transcurren las actuaciones a lo largo de una actividad; por último, el cuarto elemento se organiza en torno a intercambios comunicativos que podemos considerar como el análisis central de la actividad (Coll y Rochera, 2000; Coll et al., 2008).

Si observamos cada uno de estos elementos propuestos en el párrafo anterior, lo relevante está en los procesos comunicativos que ocurren durante una situación de enseñanza y aprendizaje, lo que tiene como base la negociación de significados y contextos discursivos que hacen posible la comunicación y la comprensión (Edwards y Mercer, 1988). Sin embargo, es necesario aclarar que dichos elementos se encuentran íntimamente relacionados con la actividad que se desarrolla a lo largo del proceso de enseñanza y aprendizaje y que, al mismo tiempo, van cambiando en la medida que transcurre la interacción. Dicho de otra forma, discurso y actividad adquieren una relevancia central para lo que entendemos como aprendizaje (Engeström, 1992; Lave, 1996).

Otro aspecto importante a considerar para el estudio de las prácticas educativas es la necesidad de definir y precisar cuáles son las prácticas que se analizan dependiendo del contexto de actividad. Si bien es cierto estas pueden ser variadas, lo relevante es que constituyan acciones recurrentes de la actividad educativa. En esta línea, trabajos desarrollados por Mauri y Barberà (2007) han puesto especial atención a las prácticas evaluativas de los aprendizajes, prácticas de especial interés dada la relevancia que pueden adquirir en el proceso de 
enseñanza y aprendizaje como mecanismo mediador entre el propio proceso y la construcción de conocimiento que realizan los estudiantes (Coll, Majós y Rochera, 2012).

Algunos de los resultados de estos trabajos han permitido identificar una serie de características de las actividades de evaluación que deben ser consideradas en la práctica evaluativa. Entre ellas, destacan las actividades de comunicación de los resultados de la evaluación, la implicación de los docentes y estudiantes en el proceso de valoración, actividades de revisión y reelaboración y la toma en consideración de la dimensión temporal de las actividades de comunicación de los resultados de la evaluación. Estos aspectos estarían en estrecha relación con la consideración de la evaluación como una actividad compleja, es decir, contempla distintos momentos y actuaciones, además de la evaluación misma (señaladas más arriba). Estas actividades están diseñadas para facilitar y guiar el paso de la regulación externa a la regulación interna de los aprendizajes (Rochera y Naranjo, 2007).

En una línea de investigación similar se encuentran los trabajos desarrollados por Pérez, R. C., Pérez, M. C., Santigosa, de la Mata, Carmona y Gallardo (2008) y Sánchez (1996). Estos trabajos comparten una serie de elementos teóricos y metodológicos, sin embargo, lo central se relaciona al reconocimiento de los recursos discursivos utilizados, en el entendido que dichos recursos permiten a los docentes favorecer la reflexión de sus prácticas a partir de la búsqueda de patrones sistemáticos y el análisis de sus funciones y consecuencias (Coll y Sánchez, 2008). En este caso, lo importante se relaciona con el estudio de los vínculos que se establecen entre lo que dice el profesor en el aula y las comprensiones previas y subsiguientes de sus alumnos (Sánchez y Rosales, 2005). Dichos trabajos consisten principalmente en descripciones del discurso educativo, tanto en términos de aquello dicen las personas, como el modo en que lo llevan a cabo (Gallardo y Cubero, 2005).

De igual forma, estos trabajos comparten una serie de marcos conceptuales con variantes propias que permiten el estudio de la interacción educativa con énfasis diferenciados, pero que en términos 
generales facilitan el análisis de la actividad que se desarrolla, el contexto y las ayudas entregadas (Sánchez y Rosales, 2005). No obstante lo anterior, es necesario señalar que los enfoques que los autores proponen a la hora de estudiar la interacción educativa son distintos, lo que responde a la naturaleza esencialmente interpretativa y constructivista de estas metodologías (Coll y Sánchez, 2008).

Por tanto, y dado el estado inicial de la investigación de los procesos de formación en docencia universitaria, una forma de abordar la actividad que ocurre en dichos escenarios es a partir del análisis de las formas en que se configura la actividad conjunta y los mecanismos de ayuda educativa que ahí se desarrollan, todo esto con el propósito de transferir determinados conocimientos al aula donde se ejerce la docencia.

\section{El papel del discurso en el estudio de las prácticas educativas}

Uno de los elementos considerados esenciales a la hora de estudiar la actividad son las estrategias discursivas que los docentes utilizan para enseñar determinados contenidos educativos. Dichas estrategias poseen características que les son propias, las que vienen determinadas por el contexto donde ocurren y por el tipo de contenido que se enseña (Cubero, 2005; Edwards y Mercer, 1988; Lemke, 1997). El énfasis está puesto en la idea que el aprendizaje se materializa a partir de la negociación de significados y contextos discursivos que hacen posible la comunicación y la comprensión (Mercer \& Coll, 1994; Mercer, 2000).

Diferentes autores plantean que las prácticas educativas se constituyen de distintas estrategias discursivas y semióticas que comprometen tanto a alumnos como docentes en la construcción de supuestos relacionados con determinados conocimientos y formas de actuación (Cole, 1985; Engeström, Miettinen \& Punamäki, 1999). En este sentido, el discurso puede considerarse el contorno que determina la actividad y, al mismo tiempo, aquello que define la construcción de significados compartidos entre los participantes. 
Esta evolución respecto del estudio de los discursos en las prácticas educativas ha posibilitado un entendimiento cada vez mayor de la forma en que estos contribuyen al proceso de enseñanza y aprendizaje. A su vez, lo anterior ha llevado a diferenciar dos aspectos importantes a considerar. El primero dice relación con aspectos más micro del discurso empleados por los participantes y el segundo, con aspectos más molares relacionados con la organización de las actividades educativas (Coll y Sánchez, 2008).

Ambos aspectos - micro y molar- pueden considerarse parte íntegra de la actividad. La diferencia entre ambos depende de la descripción que se haga de dicha actividad. Si describimos los aspectos en términos lingüísticos, estaremos hablando de análisis micro, en cambio si describimos la actividad en términos de acciones e interacciones, nos estaremos refiriendo a los aspectos molares de esa actividad. Por tanto, como lo definen sus propios autores, el análisis de la práctica debe considerar ambos elementos, en el entendido que discurso y actividad se modulan mutuamente en el transcurso de la interacción (Coll y Sánchez, 2008; Coll et al., 2008).

En consecuencia, el análisis debe considerar las propiedades del discurso o reglas discursivas, el contenido o significado de dicho discurso, y las acciones del profesor, considerando cómo estas interactúan con las de los alumnos para promover cambios o aprendizajes en estos últimos (Coll y Rochera, 2000; Coll y Sánchez, 2008). En resumen, el estudio de la práctica educativa debe incluir estos tres elementos con la finalidad de responder a los requerimientos necesarios para el abordaje de la interactividad o la relación básica entre contenido, profesor y estudiante.

Ambos aspectos pueden considerarse centrales en el análisis de la práctica educativa, a pesar de que los énfasis puedan variar en mayor o menor medida de acuerdo con la importancia que se asigne a la actividad o al discurso. A continuación, abordaremos aquellos elementos metodológicos que se desprenden del papel que juega el discurso en el estudio de la investigación sociocultural y su utilidad respecto de las potencialidades en el análisis de los procesos de formación universitaria asociados a la docencia. 


\section{Elementos metodológicos para el estudio de las prácticas educativas}

Esta línea de investigación tiene como base diferentes técnicas de análisis cualitativo, considerando como unidad de análisis los registros audiovisuales de procesos instruccionales completos. La mayoría de los trabajos en esta perspectiva estudia procesos completos debido a que ello permite incorporar al análisis los diferentes elementos de un proceso educativo (Coll et al., 2008; Mauri \& Barberà, 2007; Onrubia, Rochera y Álvarez, 1990; Sánchez y Rosales, 2005). En este mismo sentido, existe acuerdo en la necesidad de valorar la unidad completa o la dimensión temporal del proceso de enseñanza y aprendizaje, dado que la construcción de conocimiento requiere de tiempo para su elaboración (Scardamalia \& Bereiter, 2006; Schwarz, Dreyfus \& Hershkowitz, 2009).

Una vez seleccionada la unidad de análisis - una unidad didáctica completa-, es necesario identificar los episodios o segmentos de interactividad que la constituyen, que pueden describirse como una serie de actuaciones compartidas por todos los participantes (Coll y Rochera, 2000; Cubero, 2005). La existencia de estos episodios constituye un eje clave en la metodología planteada por estos autores, dado que la configuración de dichos episodios da forma a la estructura de participación o primer nivel de análisis.

Para definir o delimitar cada uno de los episodios o segmentos que constituyen una secuencia didáctica, todos los participantes del proceso instruccional deben mostrar evidencias de que aceptan las actuaciones de los otros (Onrubia et al., 1990; Rochera y Naranjo, 2007). Estas actuaciones toman sentido a partir del núcleo desde donde se configuran dichos segmentos: la relación entre profesor, alumno y contenido (Coll y Sánchez, 2008). En resumen, la definición de los segmentos responde a la actividad conjunta evidenciada a partir de los cambios que surgen a lo largo del proceso instruccional.

Para efectos de la identificación de los segmentos deben cumplirse dos condiciones. Primero, se tiene que responder a las preguntas: quién puede hacer qué, con quién lo puede hacer, y 
cuándo y cómo se puede hacer. Lo fundamental es definir la estructura de participación que surge en los diferentes momentos del proceso instruccional. Una vez hecho esto, se debe identificar la estructura del contenido o las tareas que se abordan en torno a la actividad conjunta. Por tanto, existen dos niveles que guardan estrecha relación, el primero se encuentra asociado con la estructura de participación y el segundo, con la estructura de la tarea o contenido. Ambos niveles de análisis en conjunto permiten delimitar los segmentos que aparecen, cuántos, en qué orden lo hacen, cuál es su duración y cómo evolucionan a lo largo del proceso instruccional. De la misma manera, permiten determinar patrones latentes al interior de los segmentos identificados, o dicho de otra forma, cuáles son las actuaciones que predominan y no predominan en cada uno, y en qué medida aquellas que al inicio no son predominantes, posteriormente evolucionan convirtiéndose en actuaciones predominantes (Coll y Rochera, 2000).

En el segundo nivel de análisis, y como consecuencia del primero, se encuentra la actividad discursiva que desarrollan los participantes, que se enfoca en análisis del contenido semiótico de dicha actividad. En este nivel, el objetivo es identificar cómo se construye y reconstruye el significado de la actividad a través de la interpretación de los discursos de los participantes, generando categorías analíticas que surgen de ella.

Los distintos elementos metodológicos hasta aquí referenciados han llevado a los autores a identificar diferentes formas de organización de la actividad conjunta en un proceso instruccional. Algunos trabajos muestran a partir de mapas cómo se organiza la actividad (Coll y Rochera, 2000). Estos mapas, denominados "mapas de interactividad", permiten visualizar de mejor manera cómo se estructura la participación a partir de la observación de la configuración (duración y evolución de los segmentos en la secuencia didáctica) de los segmentos de interactividad (Coll et al., 2008; Onrubia et al., 1990).

La utilidad de distinguir estas estructuras de participación a partir de los segmentos permite analizar las rutinas o configuraciones de la actividad, su duración y cómo estas se distribuyen a lo largo de 
la secuencia o proceso instruccional, lo que facilitaría la interpretación de las formas de actuar o mecanismos que puedan aparecer y que contribuyan a explicar cómo se configura la actividad (Coll et al., 2008; Onrubia et al., 1990). Ahora bien, si consideramos un proceso de formación universitario que tiene por propósito mejorar la manera cómo los docentes evalúan, el estudio de las prácticas educativas resulta interesante y de gran aporte a la comprensión de las formas que asume la actividad conjunta entre docentes formadores y docentes universitarios.

\section{Un ejemplo de análisis: el estudio de las prácticas evaluativas}

Un aspecto que resulta particularmente relevante al momento de estudiar la práctica educativa son las situaciones de evaluación. Estas instancias, en términos simples, pueden entenderse como un conjunto de procedimientos orientados a la valoración de los procesos de enseñanza y aprendizaje. No obstante lo anterior, y considerando que esta actividad promueve la reflexión y certifica los aprendizajes de los estudiantes, la mayoría de las veces, las actividades de evaluación se concretan como un proceso formal que no facilita la autorregulación de los aprendizajes que logran los estudiantes. Por esta causa, es útil entender la evaluación como un proceso complejo que sitúa al estudiante en un rol activo dentro de él (Coll y Remesal, 2009).

Un ejemplo de los trabajos desarrollados en esta línea lo podemos encontrar en el estudio de las prácticas evaluativas, donde se identifican diferentes momentos que son propios de las situaciones de evaluación (Majós y Rochera, 2010). Estos momentos se configuran como instancias donde el docente y estudiante realizan actuaciones tales como la preparación de la evaluación, la evaluación propiamente tal, la realización de correcciones y los momentos de devolución, comunicación y aprovechamiento de los resultados de la evaluación. Estos hallazgos permiten visualizar las situaciones de evaluación como procesos complejos y, a su vez, valorar la potencialidad de estos como facilitadores de la atribución de sentido y apoyos al ajuste de los significados necesarios para el aprendizaje. 
En términos generales, si analizamos un proceso instruccional donde el propósito formativo es la enseñanza de la evaluación, es necesario analizar el modo en que el docente ayuda a los estudiantes a comprender la importancia de la evaluación y su función reguladora, identificando las actuaciones que faciliten la individualización de un patrón de comportamiento dominante en el transcurso del proceso instruccional. Este aspecto contiene la idea que las ayudas asociadas a los procesos evaluativos pueden considerarse mecanismos de influencia mediadores entre la enseñanza y el aprendizaje.

Una forma de contribuir al estudio de estos procesos sería centrar el análisis en la forma en que el docente comunica a los estudiantes los resultados de la evaluación con el objetivo de observar cómo este intercambio comunicativo media la construcción de significados compartidos que favorecen el aprendizaje.

Si consideramos un corpus de datos de un proceso de perfeccionamiento universitario asociado con la enseñanza de la evaluación, lo primero sería identificar los diferentes segmentos a lo largo de un proceso instruccional. Recordemos que la configuración de estos episodios o segmentos tiene como base las actuaciones que realizan docentes y estudiantes frente a un contenido de aprendizaje. Dicho de otra manera, cada segmento o episodio constituye un conjunto de acciones compartidas que nos podría visibilizar la forma de organización del proceso instruccional completo.

La puesta en relación de los segmentos y las actuaciones predominantes y no predominantes permite identificar cómo y cuándo se ejercen las ayudas educativas, particularmente en este ejemplo, aquellas asociadas al estudio de la manera en que se comunican los resultados de la evaluación a los estudiantes. En ese caso, lo relevante es identificar en qué segmentos, cuándo y cómo se ejerce la comunicación de resultados, los que podrían asociarse eventualmente con segmentos donde predominen actuaciones vinculadas con la forma en la que el docente entrega a los estudiantes los resultados de la evaluación.

En resumen, la utilización y aplicación del estudio de la práctica educativa nos permite profundizar en el estudio de la actividad 
conjunta que ocurre en un proceso de enseñanza y aprendizaje, considerando los elementos teóricos y metodológicos referenciados en este trabajo, con sus distintos énfasis, según sean las prácticas de análisis investigadas o los distintos escenarios o niveles de enseñanza.

\section{Comentarios finales}

Existe consenso respecto de la necesidad de ampliar la investigación de la práctica educativa como alternativa metodológica para facilitar el entendimiento de los procesos de construcción de conocimiento que ocurren en diferentes niveles y escenarios educativos. Un aporte relevante desde esta perspectiva es la importancia que se da al análisis de la actividad y el discurso, poniendo especial énfasis en la actividad en sí misma. En este contexto, cabe destacar que los elementos metodológicos aquí presentados logran poner de relieve la necesidad del análisis micro y molar de la actividad educativa en diferentes niveles y escenarios.

En este contexto, son cada vez más los trabajos que abordan la actividad educativa desde el análisis de la interacción, poniendo especial atención en el ajuste de las ayudas o los mecanismos de influencia educativa que ejercen los docentes y los propios estudiantes mientras interactúan. De la misma manera, resulta cada vez más relevante analizar cómo estos mecanismos varían en función del escenario, tarea, objetivo y contenido que se pretende enseñar.

Es importante señalar que estos mecanismos se concretan a partir de las ayudas educativas y que estas pueden estar presentes tanto en situaciones que se apoyan en la actividad individual como en situaciones de colaboración. La observación de dichos mecanismos permite, en definitiva, comprender cómo se desarrollan y materializan los procesos de enseñanza y aprendizaje a partir de la observación panorámica de los diferentes componentes que constituyen la interacción, especialmente los segmentos de interactividad y las actuaciones de los participantes.

Esta línea de investigación puede considerarse una alternativa para el estudio de los procesos de formación en docencia universitaria, 
sobre todo en lo que respecta al diseño de estos programas. El estudio de las prácticas educativas y del modo en que se configura la actividad conjunta en procesos de formación docente nos permite, por un lado, conocer in situ cómo los docentes participantes aprenden y, por otro, vislumbrar cómo estos procesos de aprendizaje pueden trasladarse o transferirse a la sala de clase a través de la propia práctica de estos profesores en formación. La consideración de esto último implica la implementación de mejoras y nuevos desafíos para quienes trabajan en el área, así como también para quienes investigan este campo.

De igual manera, el estudio de los procesos de formación en educación superior con énfasis en la interacción que desarrollan sus participantes sirve al propósito de mejorar el diseño de estos programas que tienen como finalidad capacitar a los docentes en diferentes procesos asociados a la enseñanza y aprendizaje. Al observar en contextos reales cómo se desarrollan estos procesos es posible conocer la atribución de sentido necesaria para que se produzca el aprendizaje y que dicha atribución, dada su complejidad, considera tiempos y procesos que solo se pueden observar en la práctica. Dicho de otro modo, al conocer empíricamente cómo se desarrollan estos programas de formación en contextos reales es posible valorar los tiempos y duración necesarios para su implementación.

Lo anterior plantea la necesidad de considerar los programas de formación en contextos universitarios como procesos complejos que requieren de investigación desde perspectivas que superen la sola medición de resultados. En definitiva, es importante poner atención a la configuración de estos programas de formación y sus diferentes elementos, porque facilitan una mejor comprensión de su organización con la finalidad de optimizar la transferencia al aula.

\section{Referencias}

Barron, B. (2006). Interest and self-sustained learning as catalysts of development: a learning ecology perspective. Human Development, 4(49),193-224.

Cole, M. (1985). The zone of proximal development-where culture and cognition create each other. En J. Wertsch (Ed.), Culture, communication 
and cognition: Vigotskian perspectives (pp. 146-161). Cambridge, MA: Cambridge University Press.

Cole, M. (1998). Cultural psychology: a once and future discipline. Cambridge, Mass: Belknap Press of Harvard University Press.

Coll, C., Majós, T. M., y Rochera, M. J. (2012). La práctica de evaluación como contexto para el aprendizaje competente. Profesorado: Revista de Currículum y Formación del Profesorado, 1(16), 49-59.

Coll, C., Onrubia, J., y Majós, T. M. (2008). Ayudar a aprender en contextos educativos: el ejercicio de la influencia educativa y el análisis de la enseñanza. Revista de Educación, 346, 33-70.

Coll, C. y Remesal, A. (2009). Concepciones del profesorado de matemáticas acerca de las funciones de la evaluación del aprendizaje en la educación obligatoria. Infancia y Aprendizaje, 3(32), 391-404.

Coll C., y Rochera M.J. (2000). Actividad conjunta y traspaso del control en tres secuencias didácticas sobre los primeros números de la serie natural. Infancia y Aprendizaje, 4(23), 109-130.

Coll, C., y Sánchez, E. (2008). Presentación. El análisis de la interacción alumno-profesor: líneas de investigación. Revista de Educación, 346, 15-32.

Cubero, R. (2005). Elementos básicos para un constructivismo social. Avances en Psicología Latinoamericana, 1(23), 43-61.

Edwards, D. y Mercer, N. (1988). El conocimiento compartido: el desarrollo de la comprensión en el aula. Madrid: Paidós.

Engeström, Y. (1992). Interactive expertise: studies in distributed working intelligence. Helsinki: Department of Education, University of Helsinki.

Engeström, Y., Miettinen, R., \& Punamäki, R.-L. (1999). Perspectives on activity theory. Cambridge, MA: Cambridge University Press.

Ho, A., Watkins, D., \& Kelly, M. (2001). The conceptual change approach to improving teaching and learning: an evaluation of a Hong Kong staff development programme. Higher Education, 2(42), 143-169.

Kitayama, S., \& Cohen, D. (2010). Handbook of cultural Psychology. Nueva York: Guilford Press.

Lave, J. (1996). Teaching, as learning, in practice. Mind, Culture, and Activity, 3(3), 149-164.

Lemke, J. L. (1997). Aprender a hablar ciencia: lenguaje, aprendizaje y valores. Barcelona: Paidós. 
Mauri, T., \& Barberà, E. (2007). Regulating knowledge building in the classroom through the communication of learning results to pupils. Infancia y Aprendizaje, 30(4), 483-497.

Majós, T. M. y Rochera, M. J. (2010). La evaluación de los aprendizajes en la educación secundaria. En C. Coll (Coord.), Desarrollo, aprendizaje y enseñanza en la educación secundaria (pp. 155-172). Barcelona: Editorial Graó.

Mercer, N. (2000). Words and minds: how we use language to think together. London: Routledge.

Mercer, N., \& Coll, C. (1994) Teaching, learning and interaction. Madrid: Fundación Infancia y Aprendizaje.

Onrubia, J., Rochera, M. J. y Álvarez, R. C. (1990). Interactividad, mecanismos de influencia educativa y construcción del conocimiento en el aula. En C. Coll, A. Marchesi y J. Palacios (Comp.), Desarrollo psicológico y educación (pp. 437-460). Madrid: Alianza Editorial.

Prados, M. del M. y Cubero, R. (2005). Construcción del conocimiento y discurso educativo. Una aproximación al estudio del discurso de profesores y alumnos en la universidad. Avances en Psicología Latinoamericana, 1(23), 141-153.

Pérez, R. C., Pérez, M. C., Santigosa, A. S., de la Mata Benítez, M. L., Carmona, M. J. I. y Gallardo M. del M. (2008). La educación a través de su discurso. Prácticas educativas y construcción discursiva del conocimiento en el aula. Revista de Educación, 346, 71-104.

Postareff, L., Lindblom-Ylänne, S., \& Nevgi, A. (2007). The effect of pedagogical training on teaching in higher education. Teaching and Teacher Education, 5(23), 557-571.

Rochera, M. J. R. y Naranjo, M. N. (2007). Ayudar a autorregular el aprendizaje en una situación de evaluación. Electronic Journal of Research in Educational Psychology, 13(5), 805-824.

Sánchez, E. (1996). Divulgative texts as covert conversations: analysis of the communicative resources of divulgative texts. Infancia y Aprendizaje, 3(19) 85-96.

Sánchez, E. y Rosales, J. (2005). La práctica educativa. Una revisión a partir del estudio de la interaccion profesor-alumnos en el aula. Cultura y Educación, 2(17), 147-173.

Scardamalia, M., \& Bereiter, C. (2006). Knowledge building: theory, pedagogy, and technology. En K. Sawyer (Ed.), Cambridge handbook of the learning sciences (pp. 97-118). Nueva York: Cambridge University Press. 
Schwarz, B., Dreyfus, T., \& Hershkowitz, R. (2009). Transformation of knowledge through classroom interaction. New York: Routledge.

Stes, A., De Maeyer, S., Gijbels, D., \& Van Petegem, P. (2013). Effects of teachers' instructional development on students' study approaches in higher education. Studies in Higher Education, 1(38), 2-19.

Stes, A., Min-Leliveld, M., Gijbels, D., \& Van Petegem, P. (2010). The impact of instructional development in higher education: the state-of-the-art of the research. Educational Research Review, 1(5), 25-49. 